should be modified to accommodate clinical pediatric manifestations and omit those symptoms infrequently observed in children, eg unilateral headache, phonophobia. The restrictive nature of IHS adult criteria for migraine diagnosis in children is discussed in Progress in Pediatric Neurology III, PNB Publ, 1997.

Effective migraine management is reviewed from the Institute of Neurology, Queen Square, London (Goadsby PJ, Olesen J. Neurology March $1997 ; 48$ (Suppl 3):S1-S3). Acute treatment of migraine with dihydroergotamine nasal spray is reported from the University Headache Center, Moorestown, NJ. (Gallagher RM et al. Arch Neurol Dec 1996;53:12851291). Newer and experimental treatments for migraine are generally inappropriate for use in children. Analgesic or analgesic/antiemetic combinations are first-line acute treatments. Greater attention might be given to precipitating factors, including diet, school-related stress, and negative parent responses to the headache.

\title{
PARENT RESPONSES TO PEDIATRIC HEADACHE
}

Parent and child report measures of the frequency and quality of parent responses to children's recurrent headache were evaluated for 153 pediatric patients at the University of Maryland School of Medicine, Baltimore, using a 16-item scale modeled after part II of the West Haven-Yale Multidimensional Pain Inventory, which assesses patients' perceptions of others' responses to pain, and a Child Behavior Checklist, to assess parent perceptions of the child's behavioral responses. Parent-perceived negative responses (eg. ignore, leave room, frustration) were correlated with increased levels of behavior problems in adolescents. Parent affiliative or distracting responses (eg. talk or read to child, express sympathy) were associated with lower levels of functional disability in younger children. (Wall BA, Holden EW, Gladstein J. Parent responses to pediatric headache. Headache Feb 1997;37:6570). (Respond: Dr E Wayne Holden, Department of Pediatrics, University of Maryland School of Medicine, 630 West Fayette Street, Room 5-668, Baltimore, MD 21201).

COMMENT. As might be expected, parental negative response to pediatric headache is an important factor in causation of functional disability and behavioral problems associated with recurrent pain. To prevent the development of behavioral problems, parents of children 11 years of age and younger should reinforce symptom-free periods and use distractions and supportive comments in response to pain-related behavior. Parents of adolescents should be over solicitous and should avoid negative or punitive responses.

\section{HEADACHE AND CHIARI-I MALFORMATION}

The clinical features and pathogenesis of headache associated with Chiari-I malformation and syringomyelia are reviewed from the Katholieke Universiteit Leuven, Belgium. The incidence of headache reported with Chiari-I malformation has varied from 12 to $58 \%$. In a retrospective review of 62 cases of syringomyelia, only two had headache as a presenting symptom. Chiari-I headache is paroxysmal or protracted, occipital in location, aggravated by Valsalva's maneuver, effort, cough, and postural changes, and correlates with the degree of tonsillar herniation. Syringomyelia may coexist with Chiari-I malformation, but has no relation to the occurrence of headache. 
The cause of the headache is uncertain, but probably related to the tonsillar herniation. Intermittent obstructive hydrocephalus, with increased intracranial pressure, irritation of meninges at the foramen magnum, compression of upper cervical roots, and involvement of the descending nucleus of the trigeminal nerve are some of the possible causes of pain with Chiari-I malformation. (Van den Bergh R, Van Calenbergh F. Headache and headache-attacks in the Chiari-I malformation and in syringomyelia. Headache Quarterly 1997;8:15-21). (Reprints: Prof Dr Raymond Van den Bergh, Heidebergstraat 248 a B-3010 Leuven, Belgium).

COMMENT. A herniation of $5 \mathrm{~mm}$ is considered pathological and $3-5 \mathrm{~mm}$ is borderline. Minimal degrees of tonsillar herniation discovered by MRI in children with migraine type headaches is of concern and requires follow-up. Chiari-I headache may present at puberty or during first pregnancies. Hormonal changes may accentuate the tonsillar herniation and precipitate symptoms. (Progress in Pediatric Neurology II, PNB Publ, 1994;pp158-9).

\section{INTRACRANIAL HYPERTENSION}

\section{PSEUDOTUMOR CEREBRI AFTER L-THYROXINE THERAPY}

Pseudotumor cerebri in an infant treated with L-thyroxine for transient neonatal hypothyroidism is reported from the Montefiore Medical Center, Albert Einstein College of Medicine, Bronx, NY. The mother had Graves disease, treated with radioiodine ablation and thyroxine replacement. At 25 hours of life the baby was jittery and had tachycardia and respiratory distress. Thyroid function tests revealed thyrotoxicosis. Treatment with propylthiouracil was followed within 48 hours by clinical improvement, but after 3 weeks she became hypothyroid, possibly as a result of placental transfer of maternal thyroid-stimulating hormone receptor-blocking antibodies. After treatment with L-thyroxine, $10-15 \mathrm{mcg} / \mathrm{kg}$, at age 5 months she developed split sutures, a bulging fontanel, and a rapid increase in head circumference. The CT and normal CSF and electrolyte studies were compatible with pseudotumor cerebri. She recovered spontaneously with no change in the therapy. The thyroxine was discontinued at 1 year without relapse, and thyroid function, growth, head circumference, and motor development were normal. (Raghavan S, DiMartino-Nardi J, Saenger P, Linder B. Pseudotumor cerebri in an infant after L-thyroxine therapy for transient neonatal hypothyroidism. L Pediatr March 1997;130:478-480). (Reprints: Barbara Linder, MD, PhD, Division of Pediatric Endocrinology, Montefiore Medical Center, 111 East 210th Street, Bronx, NY 10467).

COMMENT. Previous reports of pseudotumor cerebri during the initiation of L-thyroxine therapy have involved children entering puberty, and hormonal mechanisms have been invoked. Apparently, this complication may also occur in infants and may require a slight modification of the dose of thyroxine.

\section{INTRACRANIAL HYPERTENSION AND CEREBRAL MALARIA}

Intracranial pressure (ICP) was monitored and cerebral perfusion pressure (CPP) calculated in 23 African children suffering from cerebral malaria and treated at the Kenya Medical Research Institute, Clinical Research Center, Kilifi, Kenya. Of 4 children with severe intracranial hypertension (ICP 